\title{
Discromatopsias congênitas e condução de veículos
}

\author{
Congenitaldyschromatopsias and driving
}

\author{
Mário Teruo Sato ${ }^{1}$ \\ Alfredo Vidal Moreira ${ }^{2}$ \\ Daniel Roncg'lio Guerra ${ }^{3}$ \\ Ana Cristina Alvarez de Carvalho ${ }^{3}$ \\ Carlos Augusto Moreira Júnior ${ }^{4}$
}

\begin{tabular}{l} 
RESUMO \\
\hline Objetivo: Apresentar a importância do uso da tabela de Ishihara para \\
o diagnóstico das discromatopsias congênitas e avaliação da incidên- \\
cia em motoristas, observando-se os erros mais comuns no teste da \\
Caixa de Cores. Além disso, abordar a relação entre discromatopsias \\
congênitas, condução de veículo e acidentes de tráfego. Métodos: \\
Foram examinados 523 motoristas, seguindo as normas da resolução \\
734/89 do Contran. Os motoristas foram submetidos ao teste de cores \\
com a Tabela de Ishihara, e os que tinham discromatopsias foram \\
submetidos ao teste da Caixa de Cores, com luzes dispostas como em \\
um semáforo. Resultados: Encontrou-se uma incidência de discro- \\
matopsias de 5,5\% (29 pacientes). Destes, 3 pacientes foram excluídos \\
do estudo, 16 possuíam grau forte e 10, o grau moderado. Desse último \\
grupo, 7 apresentaram-se com deuteranomalia e 3 com protanomalia. \\
Dos 7 com deuteranomalia, 3 apresentaram alterações no teste da Caixa \\
de Cores. Dos 3 com protanomalia, 1 paciente teve exame alterado. Dos \\
pacientes de grau forte, 14 possuíam deuteranopia e 2 protanopia, \\
sendo que todos apresentaram alterações no teste da Caixa de Cores. \\
Conclusão: Todos os pacientes com discromatopsias de grau forte e \\
metade dos pacientes com grau moderado cometeram erros no teste da \\
Caixa de Cores. Conclui-se que não haveria necessidade de ser realiza- \\
do o teste para diferenciar as cores, desde que se faça o uso da tabela \\
de Ishihara para diagnosticar as discromatopsias congênitas.
\end{tabular}

Descritores: Percepção de cores; Defeitos da visão de cores/congênito; Defeitos da visão de cores/diagnóstico; Testes de percepção de cores; Condução de veículo

\footnotetext{
${ }^{1}$ Mestre em Clínica Cirúrgica pelo Departamento de Cirurgia da Universidade Federal do Paraná. Médico Oftalmologista - Perito em Medicina do Tráfego.

${ }^{2}$ Médico Pediatra. Perito em Medicina do Tráfego.

${ }^{3}$ Acadêmico de Medicina 6o ano da Universidade Federal do Paraná.

${ }^{4}$ Diretor do Setor de Ciências da Saúde da Universidade Federal do Paraná - Professor Titular da Disciplina de Oftalmologia do Departamento de Oftalmo/Otorrino da Universidade Federal do Paraná.

Endereço para correspondência: R. Américo Vespúcio, 833 - apto 4 - bloco 1. Curitiba (PR) CEP 81010-250. E-mail: mariots@super.com.br

Recebido para publicação em 08.06.2000

Aceito em 17.07.2001
}

\section{INTRODUÇÃO}

As deficiências congênitas para as cores vermelho-verde, também chamadas de discromatopsias congênitas ou daltonismo, são defeitos hereditários ligados ao cromossoma X. Devido a esse fator, praticamente só os homens são afetados, apresentando uma incidência de aproximadamente $8 \%{ }^{(1)}$. Quanto à visão em cores, as pessoas são classificadas em monocromatas, dicromatas ou tricromatas, sendo os indivíduos normais tricromatas, pois possuem na retina os cones para o vermelho, o verde e o azul. Os que não percebem as cores são monocromatas ou acromatas. Os pacientes dicromatas, por sua vez, são classificados em deuteranope, protanope ou tritanope e os pacientes tricromatas anormais, em deuteranômalo, protanômalo e tritanômalo. * Wright em 1944 (citado por Urbano, 1979). De acordo

\footnotetext{
*Wright WD. The measurement of colour. London: Adam Hilder; 1944. 233p. apud Urbano LCV. Exames de cores na propedêutica neuro-oftalmológica. Rev Bras Oftalmol 1979;38:175-200.
} 
com a gravidade, as deficiências vermelho-verde podem ser classificadas em leve, moderada ou grave. Os graus leve e moderado (ou médio) levam o sufixo omalia. Assim, temos deuteranomalia e protanomalia. O grau grave, ou forte, leva a terminação opia. Logo, tem-se: deuteranopia e protanopia ${ }^{(2)}$. As formas mais comuns de discromatopsias congênitas são as formas deutan, e protan, com incidência variando de 7-9\%, e a mais rara, a tritan $(0,001 \%)^{(3)}$. Somente as formas congênitas protan (deficiência para o vermelho) e deutan (deficiência para o verde), também chamadas de deficiência vermelho-verde, foram consideradas neste estudo.

A literatura é confusa em relação à condução de veículos e discromatopsias congênitas. Alguns estudos afirmam que pacientes com essa deficiência não provocam mais acidentes, se comparados a pacientes normais ${ }^{(3)}$. Outros afirmam exatamente o contrário ${ }^{(4)}$. Por essas divergências e também devido à falta de normatização para visão de cores na literatura que trata da avaliação de motoristas, os autores realizaram este trabalho utilizando a Tabela de Ishihara e o teste da Caixa de Cores, de acordo com a resolução 734/89 do Contran ${ }^{(5)}$.

Este trabalho visa, então, divulgar a importância e o uso correto da Tabela de Ishihara para a classificação das discromatopsias congênitas, comparando-a com o teste da Caixa de Cores, em relação ao grau das discromatopsias. Busca também avaliar a incidência de discromatopsias congênitas na população de motoristas masculinos, em Curitiba, e os erros mais comuns cometidos por pacientes com discromatopsias congênitas no teste da Caixa de Cores. A relação entre discromatopsias congênitas, condução de veículos e acidentes de trânsito, também será discutida.

\section{MÉTODOS}

O presente estudo foi realizado prospectivamente no SEST/SENAT (capit 13 - Curitiba-PR), no período de junho de 1996 a junho de 1999.

Foram tomados os primeiros 523 prontuários de motoristas (categorias D e E) que se apresentaram para exames pré-admissionais e periódicos, em cumprimento às normas da resolução 734/89 do Contran, a qual estabelece que os candidatos a motorista devem ser submetidos ao teste de cores com tábuas pseudo-isocromáticas (Tabela de Ishihara) e à identificação das cores básicas (vermelho, amarelo e verde) na disposição do semáforo. Para este último, utilizou-se uma caixa de cores.

Todos os pacientes foram submetidos ao teste de Ishihara com 24 pranchas, foram avaliados pelo mesmo examinador. $\mathrm{O}$ teste de Ishihara foi realizado colocando-se a tabela a $75 \mathrm{~cm}$ do paciente, em ângulo reto com o eixo visual, sob iluminação natural, como determina o manual de instruções do livro ${ }^{(2)}$.

Somente os pacientes com discromatopsias congênitas diagnosticados no teste de Ishihara foram submetidos ao teste da Caixa de Cores. Esta caixa de cores foi construída em madeira, conforme o diagrama em anexo (Figura 1A-D), tendo suas partes internas pintadas de preto. Com base no projeto original do DETRAN-PR, foram realizadas algumas modificações, como a adição de mais 6 "leds" aos 3 já existentes, o que resultou em 3 "leds" vermelhos, 3 amarelos e 3 verdes, dispostos na sequiência vermelho-verde-amarelo da esquerda para a direita do examinador, totalizando 9 "leds" (Figura 1C).

Os "leds" tiveram sua luminosidade medida individualmente, diretamente na abertura pela qual o paciente vê as cores (Figura 1D), com a sala escura, por meio de um luxímetro. Essas medidas foram de 0,3 lux para o led vermelho, 0,2 lux para o amarelo e 0,15 lux para o verde. Cada "led" tem uma potência de 3 volts, sendo que todos são supridos por duas baterias do tipo AA de 1,5 volt cada. Todas essas modificações foram verificadas e aprovadas pelo DETRAN-PR.

Para a realização do exame no paciente com discromatopsia, procedeu-se da seguinte forma: o paciente era solicitado a olhar através das aberturas circulares e informar a cor que estava enxergando. Antes do exame, ele era informado que só havia 3 cores: verde, amarelo e vermelho. Os "leds" eram então acionados aleatoriamente pelo examinador, com um intervalo de 3 a 5 segundos. Caso fosse suspeitado desatenção ou nervosismo do motorista, o exame era repetido em outra consulta.

Antes de iniciado o protocolo, o método foi testado em 20 pacientes normais, que passaram sem alterações pelo teste de Ishihara. Todos reconheceram as cores dispostas na caixa. Como a caixa de cores funciona com bateria, esta era verificada pelo examinador uma vez por mês, ocasião em que eram testados os 9 leds do aparelho.

No teste de Ishihara, os motoristas eram classificados como normais para a visão das cores ou como portadores de protanomalia, protanopia, deuteranomalia ou deuteranopia.

No teste da Caixa de Cores, os motoristas com discromatopsias congênitas foram classificados como normais (não confundiam as cores ao serem submetidos aos flashes luminosos) ou com alterações (confundiam as cores). As alterações na caixa de cores foram classificadas como confusão de amarelo com vermelho, vermelho com amarelo, verde com vermelho, vermelho com verde, verde com amarelo ou amarelo com verde, sendo essas alterações imediatamente anotadas no prontuário. O motorista era considerado inapto, de acordo com a resolução vigente, se tivesse o diagnóstico de discromatopsia, verificado por meio do teste com a Tabela de Ishihara, e se fossem constatadas alterações no teste da Caixa de Cores. Caso o motorista tivesse discromatopsia, mas o teste da Caixa de Cores resultasse normal, ele era considerado aprovado.

\section{RESULTADOS}

A tabela 1 mostra os pacientes classificados quanto à intensidade da discromatopsia, de acordo com Ishihara, e os resultados no teste da Caixa de Cores, se normal ou com alteração. Dos 523 pacientes avaliados, 29 (5,5\%) apresen- 


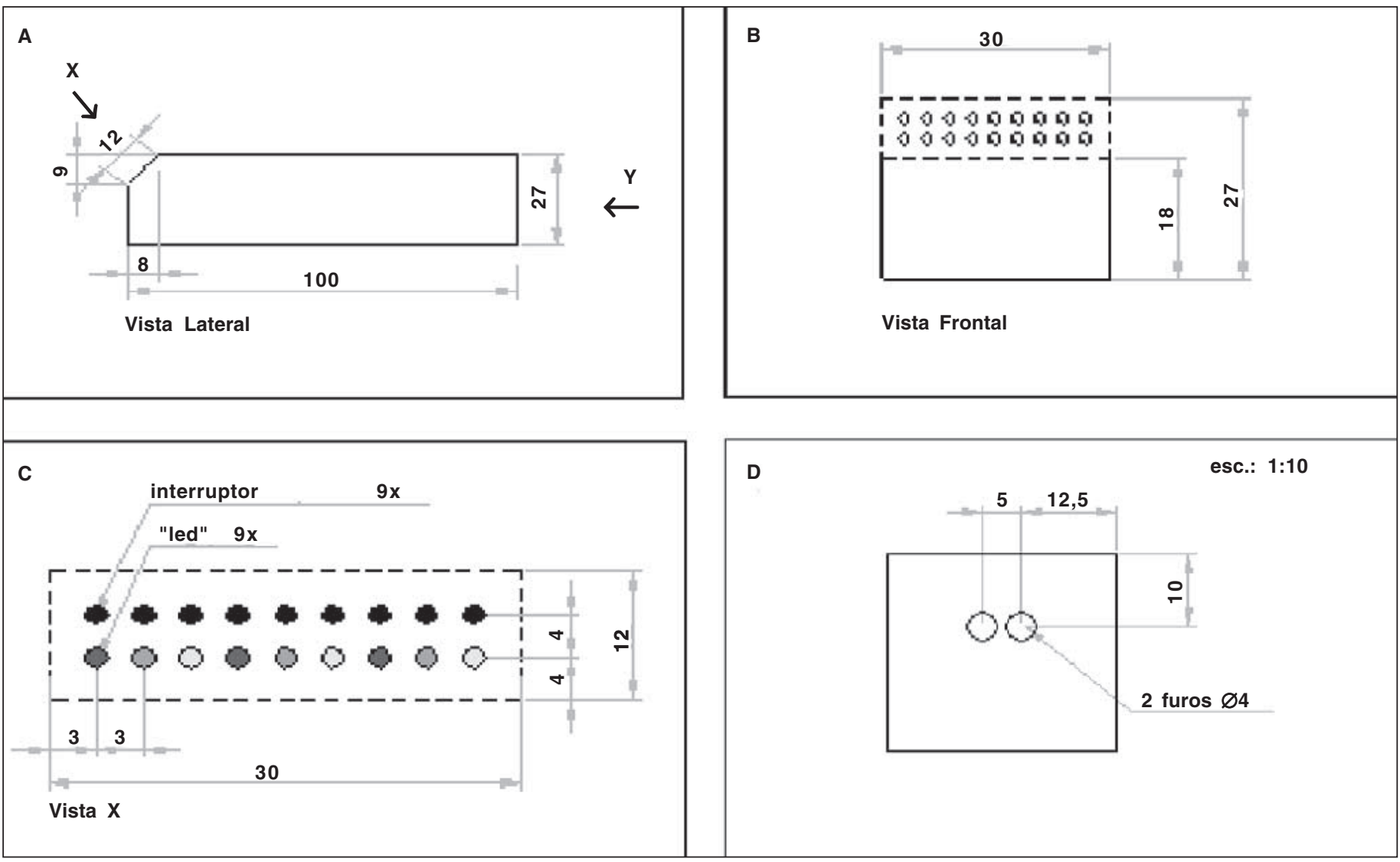

Figura 1 - Desenho esquemático da caixa de cores. Medidas em centímetros (cm). Material - madeira. A - Vista lateral da Caixa de Cores. ${ }^{\times} \rightarrow$ Vista X do examinador $\mathrm{e} \leftarrow^{\mathrm{Y}}$ Vista $\mathrm{Y}$ do paciente; B - Vista frontal da Caixa de Cores. Leds e interruptores entre as linhas interrompidas; C - Linhas interrompidas da figura 1B com maior aumento (Vista X do examinador), mostrando os interruptores e os "leds" nas cores vermelho, verde e amarelo; D - Vista frontal da Caixa de Cores (Vista Y do paciente), mostrando os círculos onde o paciente vê os "leds" acionados pelo examinador

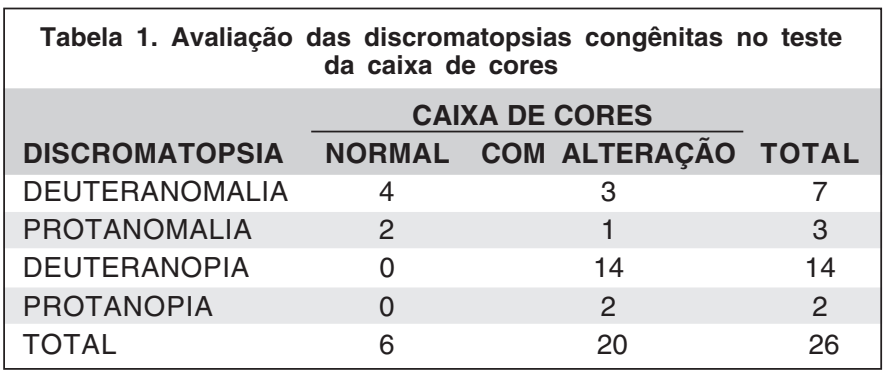

taram discromatopsia, sendo 3 excluídos do estudo, por não terem realizado o teste da Caixa de Cores, por falta de colaboração ou por não retornarem para reavaliação. Os 26 pacientes restantes foram classificados quanto ao grau de severidade da discromatopsia. Destes, 10 foram classificados como grau moderado ( 7 com deuteranomalia e 3 com protanomalia). Entre os com deuteranomalia, 4 tinham exame sem alteração no teste da Caixa de Cores e 3 com alteração, enquanto que, no grupo dos portadores de protanomalia, 2 não tinham alteração no teste da Caixa de Cores e 1 tinha exame alterado. Dezesseis pacientes foram classificados como grau forte: 14 com deute- ranopia e 2 com protanopia. Todos apresentaram alterações no teste da Caixa de Cores.

Os pacientes com alteração no teste da Caixa de Cores, foram avaliados quanto ao tipo de erro das cores. Dos 26 pacientes analisados, $20(70 \%)$ apresentaram alterações, conforme a tabela 1. Dividiu-se, então, os pacientes em grupo protan (protanomalia e protanopia), representados na tabela 2, e grupo deutan (deuteranomalia e deuteranopia), de acordo com a tabela 3 . Os erros mais freqüentes são descritos a seguir: no grupo protan, troca, em todos os testes, de amarelo por verde ou de verde para amarelo, conforme se pode observar nos dados apresentados na tabela 2 ; no grupo deutan (Tabela 3), troca de amarelo por vermelho (69\%), de amarelo por verde $(50 \%)$ e de verde para amarelo $(44 \%)$.

\begin{tabular}{|c|c|c|c|}
\hline $\begin{array}{l}\text { Cor percebida } \Rightarrow \\
\text { Cor real } \Downarrow\end{array}$ & VERMELHO & VERDE & AMARELO \\
\hline VERMELHO & - & & \\
\hline VERDE & & - & $2(100 \%)$ \\
\hline AMARELO & & $2(100 \%)$ & - \\
\hline
\end{tabular}




\begin{tabular}{|c|c|c|c|}
\hline \multicolumn{4}{|c|}{$\begin{array}{l}\text { Tabela 3. Tipo de erro das cores do grupo deutan no teste da } \\
\text { caixa de cores }(\mathrm{N}=17)\end{array}$} \\
\hline $\begin{array}{l}\text { Cor percebida } \Rightarrow \\
\text { Cor real } \Downarrow\end{array}$ & VERMELHO & VERDE & AMARELO \\
\hline VERMELHO & - & $3(19 \%)$ & $1(6 \%)$ \\
\hline VERDE & $5(31 \%)$ & - & 7 (44\%) \\
\hline AMARELO & $11(69 \%)$ & $8(50 \%)$ & - \\
\hline
\end{tabular}

\section{DISCUSSÃO}

A incidência de discromatopsia em 523 motoristas urbanos de Curitiba foi de 5,5\%, valor semelhante ao encontrado em outro estudo, em que foram avaliados 3285 pacientes masculinos em uma amostragem feita em Roma, na qual se verificou incidência de 6\%. Em geral, a incidência varia de 7 a $9 \%$ em caucasianos $^{(6)}$. Essas incidências variam de acordo com os grupos raciais: em japoneses a proporção aceitável é de $5 \%$ e em aborígenes australianos, cerca de $3 \%$. Verificou-se que 7,7\% dos pacientes possuem discromatopsia na região de Birmingham (Inglaterra), sendo que 5,4\% eram de deutan e $2,3 \%$ de protan. Zehnder encontrou deficiência vermelhoverde que varia entre 8 a $10 \%$ em homens e é de $0,4 \%$ em mulheres ${ }^{(3)}$. Os defeitos tritan são mais raros e de herança dominante. A incidência de tritanopia não é maior do que 1:13.000 e um número aproximadamente igual de homens e mulheres é afetado $^{(3)}$.

Vários trabalhos demonstram que o teste de Ishihara é o mais conhecido, de mais fácil aplicação e o método mais eficiente e sensível na detecção da deficiência vermelho-verde ${ }^{(7-8)}$. Para o uso da Tabela de Ishihara, algumas condições devem ser observadas, como: usá-la na distância de $75 \mathrm{~cm}$ dos olhos, com luz natural ${ }^{(2,6)}$ ou semelhante à natural, como, por exemplo, as lâmpadas de MacBeth Easel, que oferecem luz natural de aproximadamente 350-400 lx a $1 \mathrm{~m}$ da fonte ${ }^{(7)}$.

Os nossos resultados demonstram que, mesmo em pacientes com a forma moderada de daltonismo, houve alteração no teste da Caixa de Cores em aproximadamente metade dos examinados. As confusões de cores mais comumente encontradas foram: amarelo por vermelho (69\%) no grupo deutan, e amarelo por verde ou verde por amarelo, no grupo protan. Todos os pacientes deste grupo apresentaram tais alterações, resultado semelhante ao encontrado em teste das luzes nos semáforos ${ }^{(9)}$. Um fato importante observado é que houve grande confusão de verde por vermelho $(31 \%)$ nos deuteranopes, o que é muito perigoso no trânsito, visto que o indivíduo poderia confundir um sinal fechado com um aberto. Esse resultado seria esperado no grupo protan, mas conclusões não podem ser tiradas, devido à amostra muito reduzida nesse grupo. O teste na Caixa de Cores é similar ao da Lanterna de Giles-Archer, em que luzes são dispostas a uma distância de 5 metros, nas cores vermelha, verde, amarela e branca ${ }^{(10)}$. O exame no grupo de motoristas da região urbana de Curitiba demonstrou também que há correlação de $100 \%$ em relação ao grau forte de discromatopsia e confusão de cores. Já nos graus moderados, essa alteração foi de $50 \%$, o que mostra a estreita relação entre a gravidade e a confusão de cores. Shirley e Gauthier demonstraram, em teste com semáforos de baixa intensidade, que o erro foi de $10 \%$ no grupo protan e de $5,2 \%$ no deutan, ao passo que, aumentando-se a intensidade das luzes e utilizando-se símbolos nos semáforos (quadrado para vermelho, redondo para verde e forma de diamante para o amarelo), nenhum dos pacientes cometeu erros, concluindose que motoristas dicromatas poderiam dirigir com maior segurança se fosse aumentado o brilho do semáforo e usadas formas diferentes para o mesmo ${ }^{(9)}$. A diferença de resultados entre o presente estudo e o de Shirley e Gauthier, deve-se provavelmente ao fato de que as luzes do semáforo são maiores, mais intensas e de visualização mais fácil do que os leds da caixa de cores. Usando filtros de vidro, de acordo com os critérios de Judd, que visam a padronizar a identificação das cores por pessoas com discromatopsias, $65 \%$ dos pacientes com deficiência vermelho-verde confundem a cor verde, o que leva à conclusão de que a cor verde não é segura. $\mathrm{O}$ amarelo também foi confundido por grande parte dos pacientes, que o identificavam como vermelho, sendo também considerado pouco seguro. O pior desempenho nessas avaliações, contudo, coube aos protanopes. Os autores concluem que, se os semáforos tivessem somente duas cores (vermelho e verde), estas seriam mais facilmente identificáveis pelos dicromatas ${ }^{(11)}$.

A resolução 734/89 do Contran, em que se baseou este trabalho, exige que a avaliação oftalmológica seja feita usando-se tábuas pseudo-isocromáticas (Tabela de Ishihara), assim como seja realizada a posterior identificação das cores vermelha, amarela e verde (teste da Caixa de Cores, preconizado pelo DETRAN-PR). Quanto à resolução 80.98, atualmente em vigor, não há menção ao teste para cores com a Tabela de Ishihara, mas apenas a recomendação de que o paciente deve ser capaz de identificar as cores verde, vermelha e amarela, o que a torna inespecífica quanto à classificação das discromatopsias congênitas ${ }^{(12)}$.

Acerca da relação entre discromatopsias congênitas e acidentes de trânsito, os resultados têm sido contraditórios, já que muitos autores afirmam que os pacientes com daltonismo podem dirigir sem perigo, com desempenho semelhante ao de pessoas sem dificuldades para cores. A seqüência das luzes é normatizada obedecendo à ordem vermelho, amarelo e verde e, por esse motivo, os acidentes causados por daltônicos são insignificantes $^{(13)}$. Quando foram comparados 149 motoristas de ônibus com deficiência para visão das cores com 149 motoristas normais que usaram a mesma rota, não foram encontradas diferenças significativas de acidentes de trânsito entre os dois grupos ${ }^{(14)}$. Isso sugere que os pacientes com discromatopsias congênitas não apresentam riscos maiores ao trânsito, e que eles são confiáveis mesmo tendo carteiras C, D ou $\mathrm{E}^{(4)}$. Em uma revisão da literatura, não se encontrou associação entre pacientes com discromatopsias congênitas e acidentes 
de tráfego ou a diminuição do desempenho da condução( ${ }^{(5)}$. Outros trabalhos, no entanto, salientam as dificuldades pelas quais passam os pacientes com daltonismo. Em testes de direção aplicados em 6 pacientes deutan, observou-se que algumas dessas pessoas levavam até 100 segundos para definir a cor do semáforo ${ }^{(14)}$. Foram feitos testes em vários pacientes com protanomalia e com protanopia e constatou-se que todos tiveram dificuldades para diferenciar o amarelo do vermelho nos semáforos. Esses mesmos pacientes conseguiram identificar a diferença entre o vermelho, o amarelo e/ou o verde, quando as luzes dos semáforos eram bem destacadas e intensas. Devido à confusão entre vermelho e amarelo, sugeriu-se a retirada da cor amarela dos semáforos, mantendo-se as cores verde e vermelha. **Spiecker em 1964 (citado por Gramberg-Danielsen, 1966).

Pacientes protanopes necessitam de aproximadamente quatro vezes mais intensidade luminosa que o normal para ver o vermelho do semáforo.

Além disso, os protans parecem estar quase duas vezes mais envolvidos em colisões traseiras, se comparados a pessoas normais para visão de cores $(\mathrm{p}<0,0005)$, ou aos deutans $(p<0,002)^{(6)}$. Estudos têm demonstrado que os pacientes com discromatopsias congênitas demoram mais para reconhecer as cores do que as pessoas normais e que, ao fazê-lo, cometem duas vezes mais erros. Os pacientes daltônicos que se envolvem em acidentes de trânsito não informam voluntariamente, nem são inquiridos, sobre sua condição de deficiência visual. Por essas razões, os acidentes de trânsito, principalmente em cruzamentos, envolvendo motoristas com discromatopsias, não têm relevância estatística ${ }^{(15)}$. Mudanças na forma, na cor e no desenho dos semáforos foram sugeridas para ajudar esses motoristas $^{(16)}$.

Alguns autores defendem que pacientes com problemas de visão para cores devem ser liberados somente para as categorias A e B de motoristas (categorias não-remuneradas), medida que já está sendo adotada em vários estados ameri$\operatorname{canos}^{(17) \text {. }}$

\section{CONCLUSÕES}

O teste da Caixa de Cores mostrou alterações proporcionais à gravidade das discromatopsias congênitas, ou seja, os pacientes considerados possuidores de grau forte erraram mais que os da forma moderada. Os erros mais freqüentes foram a troca de amarelo por verde no grupo protan, e de amarelo por vermelho no grupo deutan.

A resolução 734/89 do Contran é mais completa em relação à resolução atual 80/98, porque inclui o teste com tabelas pseudo-isocromáticas.

\footnotetext{
** Spiecker HD. Praktische Untersuchungen über das Verhalten Farbsinngestörter im Strabenverkehr (1964) apud Gramberg-Danielsen B. The time-threshold of color perception in colorblind drivers. Klin Monatsbl Augenheilkd 1966;149:745-50.
}

Os resultados deste trabalho sugerem que os pacientes com discromatopsias congênitas têm maiores dificuldades na condução veicular e, conseqüentemente, são passíveis de maior envolvimento em acidentes de trânsito, principalmente nos cruzamentos.

Os nossos resultados demonstram que todos os pacientes com discromatopsias congênitas de grau severo e metade dos pacientes com o grau moderado, cometeram erros no teste da Caixa de Cores. Conclui-se que não há necessidade de realizar o teste para diferenciar as cores desde que se faça o uso da Tabela de Ishihara para diagnosticar as discromatopsias congênitas.

\section{ABSTRACT}

Purpose: To determine the importance of the Ishihara test for the diagnosis of congenital dyschromatopsias and evaluate their incidence in a population of drivers, to assess the most common mistakes in the Color Box test and to study the relationship between dyschromatopsia, driving and traffic accidents. Methods: 523 drivers were examinated, in conformity with Contran resolution 734/89. The drivers were submitted to the color test with Ishihara plates, and those who had dyschromatopsia were submitted to the Color Box test (red, yellow and green), with LEDs as used in a traffic light. Results: An incidence of 5.5\% (29 patients) of dyschromatopsias was observed. Of these patients, 3 were excluded from the study, 16 had severe disease and 10 had mild disease. In the latter group, 7 presented deuteranomaly and 3 protanomaly. Of those with deuteranomaly, 3 presented alterations in the Color Box test and 4 did not. Of those with protanomaly, 1 patient had abnormal results and 2 were normal. Of the patients with severe disease, 14 presented deuteranopia and 2 protanopia. All of them had an abnormal Color Box test. Conclusion: All patients with severe disease and half of those with moderate disease, made mistakes in the Color Box test. This study concludes that the Color Box test does not need to be performed in those patients who had the Ishihara Plate test done to diagnose congenital dyschromatopsias.

Keywords: Color perception; Color vision defects/congenital; Color vision defects/diagnosis; Color perception tests; Automobile driving

\section{REFERÊNCIAS}

1. Whillans MG. Colour-blind drivers' perception of traffic signals. Can Med Assoc J 1983;128:1187-9.

2. Ishihara S. The series of plates designed as a test for colour-deficiency. 24 plates ed. Tokyo: Kanehara; 1978.

3. Zehnder E. Performance of drivers with impaired color sense in traffic. Schweiz Med Wochenschr 1971;101:530-7.

4. Whillans MG, Allen MJ. Color defective drivers and safety. Optom Vis Sci 1992;69:463-6.

5. Brasil. Conselho Nacional de Trânsito (Contran) Resolução 734, de 31 de julho de 1989. Reformula a Resolução no. 670/87, que estabelece normas para a formação de condutores de veículos automotores, modelo de carteira nacio- 
nal de habilitação e dá outras providências. Diário Oficial da República Federativa do Brasil, Brasília, seção 1, n.150, p.13340-53, 8 ago. 1989.

6. Malaspina P, Ciminelli BM, Pelosi E, Santolamazza P, Modiano G, Santillo $\mathrm{C}$, et al. Colour blindness distribution in the male population of Rome. Hum Hered 1986;36:263-5

7. Birch J. Efficiency of the Ishihara test for identifying red-green colour deficiency. Opthalmic Physiol Opt 1997;17:403-8.

8. Linksz A. Color vision tests in clinical practice. Trans Am Acad Ophthalmol Otolaryngol 1971;75:1078-90.

9. Shirley SY, Gauthier RJ. Recognition of coloured lights by colour defective individuals. Can J Ophthalmol 1968;3:244-53.

10. Urbano LCV. Exames de cores na propedêutica neuro-oftalmológica. Rev Bras Oftalmol 1979;38:175-200.

11. Heath GG, Schmidt I. Signal color recognition in color defective observers. Am J Optom Arch Am Acad Optom 1959;36:421-37.
12. Brasil. Conselho Nacional de Trânsito (Contran). Resolução no. 80, de 19 de novembro de 1998. Altera os anexos I e II da resolução no. 51/98 - Contram, que dispõe sobre os exames de aptidão física e mental e os exames de avaliação psicológica. Diário Oficial da República Federativa do Brasil, Brasília, seção 1, n.223, p.21-24, 20 nov. 1998.

13. Keeney AH. Ophthalmic pathology in driver limitation. Trans Am Acad Ophthalmol Otolaryngol 1968;72:737-40.

14. Norman LG. Medical aspects of road safety. Lancet 1960;1:1039-45.

15. Whillans MG. Colour-blind drivers and traffic signals. Can Med Assoc J 1993; $149: 1630$

16. Waugh D. Why are we blind to the colour blind? Can Med Assoc J 1993; 148:442.

17. Light WO, Burg FD, Stock MS, Douglass JM. Guidelines for examining the driver with visual difficulty. Am J Ophthalm 1970;69:1019-22.

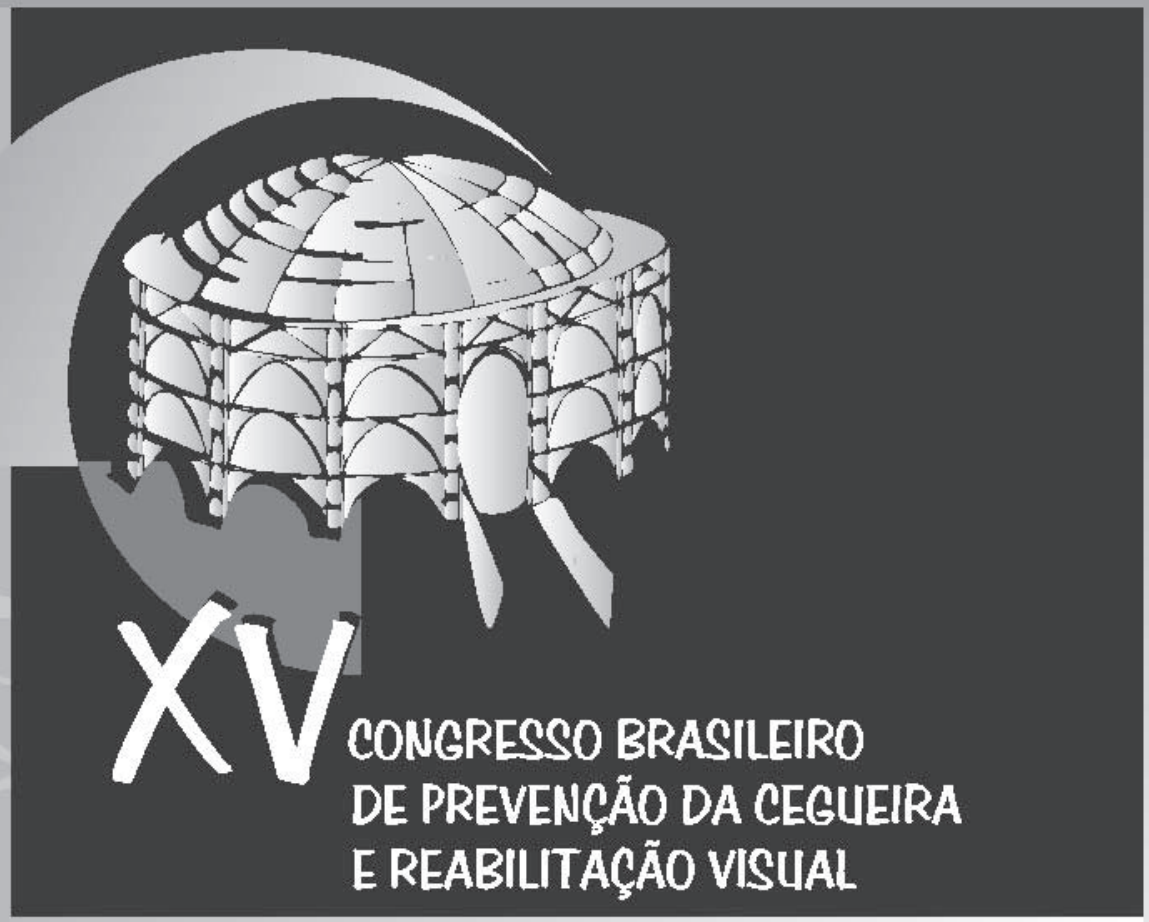

\section{1 de agosto a 3 de setembro de 2002 EXPOTRADE CURITIBA}

\section{PARTICIPE}

\section{Data limite para ehvio} de femas livres, pôstores e easos clínicos: 15/04/02

Dafa limite para envio de vídeos: 17/06/02 Al sarto, 1348 \& 1110 